\title{
RESEARCH ON REMOULDING RURAL PUBLIC SPACES IN THE MOUNTAINOUS AREAS OF WESTERN CHINA FOR ECO-MIGRATION: A CASE STUDY OF A RURAL RESETTLEMENT AREA FOR ECO-MIGRATION IN THE JINYUN MOUNTAIN, CHONGQING
}

\author{
FANGJING LUO \& YIFAN TANG \\ Chongqing University, China
}

\begin{abstract}
In China, the ecologically fragile areas and poor rural areas have highly consistent distribution characteristics and mainly distribute in the western mountainous areas. In such rural areas, due to the lack of awareness of reasonable development by villagers, the problems of ecological environment destruction and economic sustainable development are becoming more and more serious. In order to solve this problem, China began to explore eco-migration, which played an important role in improving the regional, ecological environment and lifting villagers out of poverty. Considering the factors such as the migrants' long-term living habits and regional culture, most of the receiving areas for migrants have been chosen in areas close to the relocated countryside. In this context, we focused on the current design of the new public space for migrants. However, we found that most of the existing designs apply the urban model, which made the villagers feel they had no sense of belonging. This study took the rural resettlement area for eco-migration in the mountainous area of Chongqing as an example and applied a new typological method of rural public space. Firstly, the public spaces of mountainous rural areas similar to the relocated rural area were analysed from the historical forms, and the prototypes of these public spaces were extracted in three layers: plane pattern, structural organization, decoration and function. Furthermore, these prototypes were transformed into new patterns according to the life-style that the villagers wanted. Ultimately, the various types of transformed public spaces were compounded and optimized to form the public space system of new rural resettlement. In principle, a new design model for the public space of rural resettlement that can better meet the material and spiritual needs of migrants was discovered, and with the aim to provide a reference for the future public space design of eco-migration rural resettlement.
\end{abstract}

Keywords: eco-migration, rural public space, typology.

\section{INTRODUCTION}

The distribution characteristics of poverty areas and ecologically fragile areas are highly correlated in China, and they also have a high degree of consistency in geographic distribution [1]. A total of $74 \%$ of the population in ecologically fragile areas live in poor rural areas, accounting for about $81 \%$ of the total poor population [2]. In these areas, the stability of the ecosystem is poor and its ability to resist interference is weak. However, the villagers in these regions are often forced by the pressure of survival, in exchange for short-term economic development at the expense of the ecological environment. This makes the already fragile ecological environment increasingly deteriorating under unreasonable development, which further makes the ecological and economic composite system more difficult to recover [3]. This situation is very typical in the mountainous areas of western China because its forest has been destroyed, soil erosion and flood disasters are more serious [4]. Based on the current status of such regions, China began to carry out eco-migration. Eco-migration is defined as migration activities due to the deterioration of the ecological environment or to improve and protect the ecological environment [5]. 


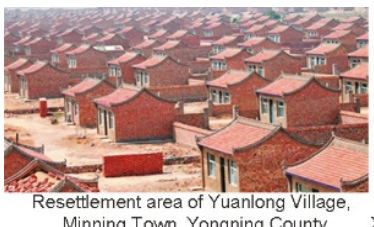

Minning Town, Yongning County

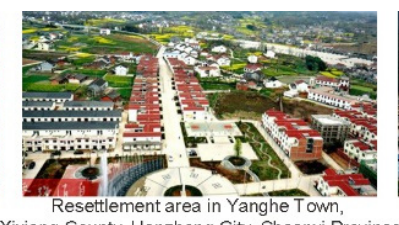

Xixiang County, Hanzhong City, Shaanxi Province

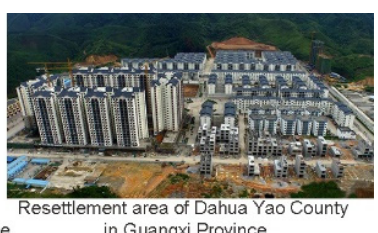

in Guangxi Province

Figure 1: The current situation of an eco-migration resettlement area in China.

This move has been implemented in China for more than 20 years, and its goals have changed from eliminating poverty and improving ecology to achieving multiple goals such as ecology, rural revitalization, and sustainable development [1]. Taking into account the long-term living habits and regional culture of the migrants, the eco-migration resettlement area is closer to where they originally lived. This will help to inherit the culture of rural areas and help migrants adapt to the new environment [6]. These needs constitute a difficult challenge, that is, how to build the rural resettlement area for eco-migration.

During the construction of the eco-migration resettlement area, the construction of rural public space in the context of globalization do not consider the scale and environment, and it is constructed in a templated modern construction method, which make the public space lack of regional characteristics, and make the migrants unable to adapt to the new surroundings. It is mainly reflected in these aspects: the courtyard is no longer flexible, and even many resettlement areas do not even have a courtyard; the traditional lanes conform to the change of terrain and harmonizes with the original natural texture. But now, the land in most resettlement areas is directly levelled, and the lanes are therefore excessively unified; The traditional culture contained in rural public spaces is represented by a series of monotonous symbol; the decorative materials of rural public spaces are all replaced by modern materials such as concrete, etc. (as shown in Fig. 1). For migrants, the public space is the carrier of their various activities. The current construction method will inevitably lead to the rural public space deviating from the original rustic atmosphere and departing from the traditional spatial pattern and public activity methods. These practices make it difficult for migrants to integrate into the new public space, fail to meet their needs for the quality of public space, and make them unable to find a sense of belonging.

Based on this situation, discussing how to remould the public space of the rural resettlement area for eco-migration is indeed an important project, but it is still a research gap at present. From a sociological point of view, rural public space is an important spiritual place for Chinese villagers. The scene of social activities in it has been constantly changing, but under the connection of geography and blood, rural public space has always maintained a stable mode of interpersonal communication. In addition, it can also give subjective meaning to the crowds through scene characteristics and obvious boundaries, which enables people to obtain a unified action force to form a specific social order. From the perspective of design, rural public space shows regional characteristics in terms of shape, structure, materials, functions, etc. Many Chinese scholars have explored the design methods of rural public spaces from a regional perspective. It is worth noting that most of these studies only considered a certain element of public space and lack a systematic design method, and it is difficult for us to refer to them. In addition, there are some studies that do not consider the characteristics of villages and the willingness of villagers. Therefore, we must avoid these problems when conducting research on the remoulding of public spaces in rural resettlement area for eco-migration, and discover a systematic design method suitable for eco-migration resettlement areas to maintain the rural emotion of migrants and promote the rural spirit. 
This paper takes the rural resettlement area for eco-migration in the Jinyun Mountain as a sample, and applies a new typological method applicable to the public space of the resettlement area. This study aims to provide a new pattern, which is commonly used for public space remoulding in mountainous eco-migration resettlement areas in western China. The public space under this pattern can meet the material and spiritual needs of migrants. And by this way, the gap in this research field can be filled.

\section{RESEARCH OBJECTS AND METHODS}

\subsection{The rural resettlement area for eco-migration in the Jinyun Mountain, Chongqing}

Chongqing is located in the mountainous region of western China. Affected by the terrain, about $1 / 4$ of the rural areas have poor construction conditions. In 2007, in order to develop the economy and protect the ecology, Chongqing put forward the goal of implementing ecological or poverty migration of 350,000 people. This also belongs to the first round of eco-migration project initiated by the Chinese government. By the end of 2012, Chongqing had more than 500,000 migrants for ecology.

As an important ecological barrier in Chongqing, parts of Jinyun Mountain were listed as national nature reserves in 2001. Jinyun Mountain has rich plant resources, containing a large number of endangered species and endemic species. Therefore, Jinyun Mountain has the reputation of "plant material gene bank". However, villagers in Jinyun Mountain Nature Reserve destroy woodlands for building houses to develop agriculture and tourism. This behavior has caused unprecedented damage to the ecological environment of Jinyun Mountain. In order to protect the ecological environment and resources of Jinyun Mountain, the Chinese government began to guide the ecological migration of protected areas in an orderly manner in 2018. The immigrations mainly include 172 households of Jinyun Village and Beiquan village in the reserve. According to the villagers' wishes, the first-phase project of the resettlement area is located on the west side of the protected area, and now belongs to Bolin Village of Chengjiang Town with a total area of 24.74 ha. The second-phase project is next to Bolin Village and is now located in Wuyi Village, Chengjiang Town. The design object is the public space of the first-phase project located in the Bolin village. This will provide guidance for the construction of the second-phase and provide a paradigm for the remoulding of public spaces in similar eco-migration resettlement areas in the future.

\subsection{A new typological method}

Before initiating this project, we must first know that the public spaces in the eco-migration resettlement area can make the rural meaning lasting. The morphological characteristics they present are compatible with the long-term survival requirements of migrants. And they can fit many aspects of migrants' life, thinking and behaviour. That is, as far as Alan Colquhoun is concerned, the changing spatial system is composed of historical remains circulated in the forms of fragments. These forms continue the meaning of the spatial system, and their significance do not depend on the changeless organization of a specific time or place [7]. These forms are types, and the conversion mechanism for conveying meaning is the method of typology. The second is how to run this conversion mechanism. Quatremère de Quincy compares "type" with "model", pointing out that typology is not a strict imitation and copy of things, but traces the origin of the fixed content of the building, and runs through the design guidelines [8]. Giulio Carlo Argan explained on this basis, pointing out that the type is the consensus and identification of the "form". These forms were established through 
comparison and augment and evolved in history. Respect for type allows historical meaning to be flexibly restored [9]. This shows that typology can be used as a metaphysical epistemology to explain the application of traditional village public space types to the new village public space under the current development needs. It is also an operational methodology that can be adapted to development requirements on the premise of continuing the meaning of the countryside. Therefore, the typology can be the correct way to guide the reconstruction of the public space in the eco-migration resettlement area.

As a design method, typology also has many successful cases, which provide methodological support for our subsequent research. Rob Krier summarized urban space prototypes and used the reduction results of these prototypes in a specific environment to guide urban design [10]; According to the construction work procedures (plan, structural system and surface treatment), Argan divided the building type into three layers: spatial configuration, structural elements and decorative elements, and he used this method for architectural design [11]; Rossi pointed out that type is a combination of a lifestyle and a form. The typology-guided design first extracts and analyses the prototypes that adapt to basic life needs and unique lifestyles in the historical context, and then transforms and reorganizes these prototypes in specific scenarios. This process is close to Jung's concept of "collective unconsciousness", which refers to the group psychological accumulation formed by the traditional experience passed on from generation to generation [12]. Combining these researches, this study firstly deduced the method of hierarchical division of building types, and divided the hierarchical layers of public space elements into plane shape, structural organization, decoration and function. Each single element is determined by these three layers of attributes. Secondly, considering the composite nature of public space, taking "layering + superimposition" as the basic idea, the single element is generated by superimposing the various types of layers being translated, and the public space system is generated by superimposing each single element. Finally, the logic of public space design for eco-migration resettlement areas guided by typology is formed. That is the following four stages: basic survey-induction and discrimination of prototypes-translation of single element-generation of public space system. The specific technical route is indicated by Fig. 2. The specific description will be given in combination with the public space design practice of Jinyun Mountain eco-migration resettlement area.

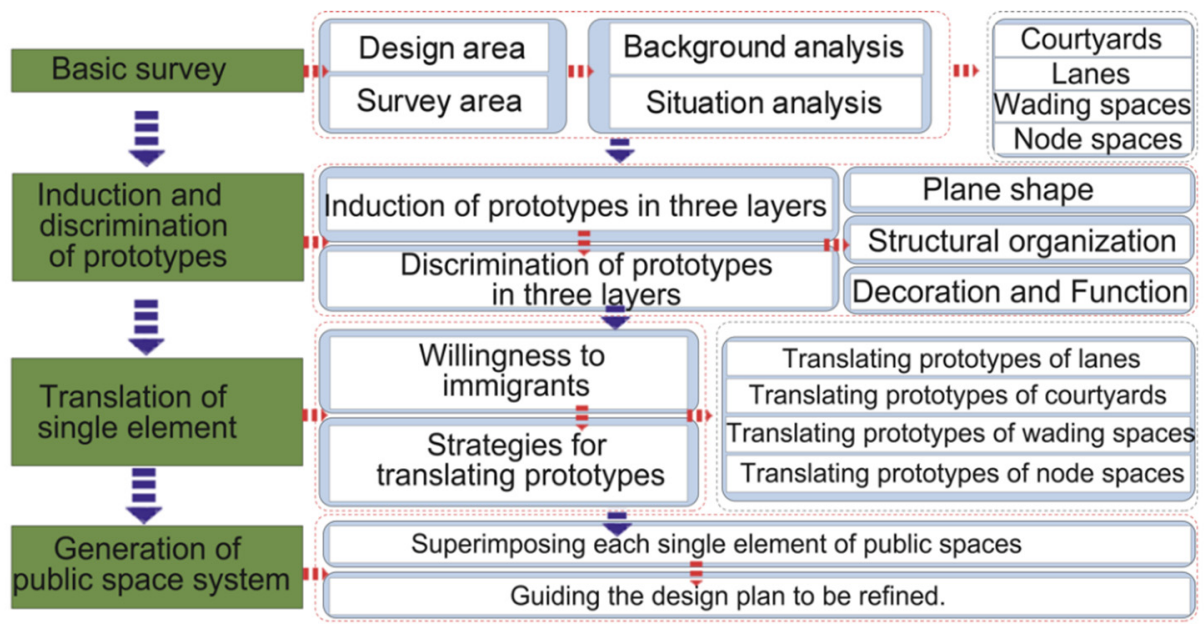

Figure 2: Technical route of village public space design using a typology method. 


\section{DESIGN PRACTICE OF PUBLIC SPACE IN RURAL ECO-MIGRATION RESETTLEMENT AREA OF JINYUN MOUNTAIN}

\subsection{Basic survey}

This design is aimed at the first-phase pilot project of Jinyun Mountain eco-migration resettlement area. The resettlement area is located in Bolin Village, Chengjiang Town. Migrants mainly come from Jinyun Village and Beiquan Village, both of which are within nature reserves. After ecological migration, the forest in the nature reserve will be restored, and the ecological environment will be gradually improved. Based on considerations of ecological priority, respect for public opinion and convenient transportation, the first-phase resettlement area was selected in Bolin Village and the second-phase resettlement area was selected in Beiquan Village. These two areas do not belong to the nature reserve, but are close to the relocated villages, and their ecological sensitivity is low. Indigenous people have moved out in large numbers and left enough land to meet the needs of migrants with less damage to the ecological environment. Moreover, the receiving areas and the relocated villages all belong to the southern part of Chengjiang Town, and the social status, lifestyle, and behaviour of the all villagers are similar, which can provide migrants with a sense of belonging. Hence, in the induction and discrimination of prototypes, the four villages are studied as similar systems (as shown in Fig. 3). The public spaces in the area mainly include courtyard, lane, wading space and node space. Most of them still follow the traditional life experience and construction wisdom to retain the traditional space form, engineering technology and lifestyle [13]. However, in recent years, the promotion of urbanization has caused the gradual decline of traditional public spaces. Some newly built public spaces try to apply innovative technologies, but they have become out of step with the atmosphere of the countryside.

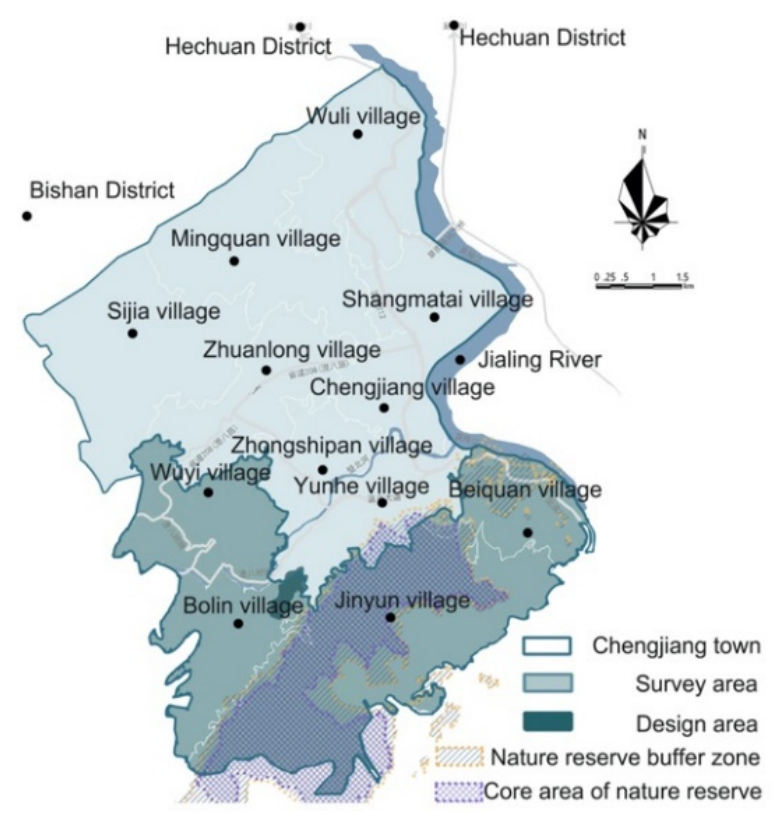

Figure 3: Location diagram of survey and design area. 


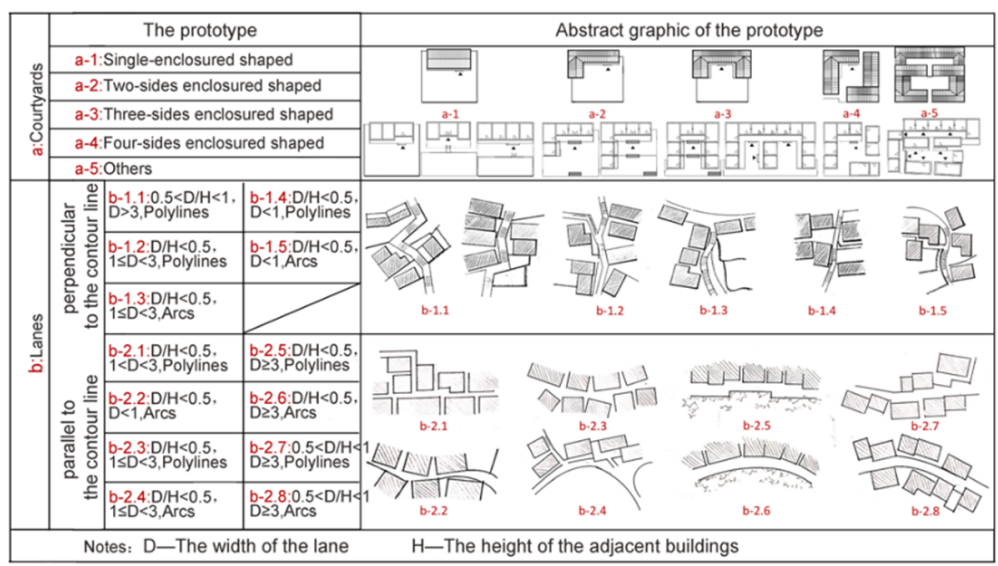

Figure 4: The prototype of the courtyards and lanes.

\subsection{Induction and discrimination of prototypes}

The prototype is summarized and identified from three layers: plane shape, structure organization, decoration and function. Each layer involves four public space elements: courtyard, lane, wading space and node space.

\subsubsection{Plane shape}

The courtyards are used for public activities in rural areas of China, and their plane shape is related to the household income and the number of family members. When the family population is small and the economic income is low, the courtyards are mostly single-sided enclosed. With the increase of economic income and the growth of the family population, the courtyards of two-sides enclosed, three-sides enclosed, and four-sides enclosed have gradually increased. The shape of these courtyards also depends on the terrain. Under the influence of topography, these courtyards can be divided into several terraces to be enlarged. In addition, Chinese people often stress the harmony between Yin and Yang. Under this concept, the baokan in the mountainous area of Chongqing, as a transition space between indoor and outdoor space, has becomes the main place for public activities in rainy days.

The plane shape of the lanes is mainly related to the mountain terrain. The villagers built mountain lanes in the respect and adaptation of the mountains, and treated the mountains slightly, so that the lanes were integrated into the mountain in a way parallel or perpendicular to the contour. In addition, villagers usually go out on foot, and traditional lanes also consider human scale. Therefore, the overall shape of lanes varies from one place to another one, and the specific turns and even the width are determined according to the terrain and daily life habits (as shown in Fig. 4).

The plane shape of the wading space is diverse. Since ancient times, China has been paying attention to the use of water for "increasing benefits and avoiding harm". Therefore, it is necessary to connect water sources when drilling wells and digging ponds, while avoiding flood disasters. Villagers also connect rivers and ponds by digging channels to form a continuous water system. The water system is usually close to the edge of the farmland or building to facilitate water intake. Therefore, the flow direction of water is consistent with the edges of farmland and buildings, which also makes the wading space appear regular arc or polyline. 
The nodes of the village mainly include the village entrance and vacant land. The village entrance is an important place to greet and bid farewell to friends. It's always without obvious boundaries but marked by big trees, stone monuments, etc. The vacant land is mainly formed by the cross-expansion of lanes or wading spaces. They are free forms but can be identified. For these places, villagers often choose nicknames of specific elements in the form of dialects, such as "Shuzibaba". These dialects represent the unique spatial concept in the hearts of villagers in this area [14].

\subsubsection{Structural organization}

The structural organization of courtyards, lanes, wading spaces and nodes in mountainous rural areas is related to the terrain. When the courtyard layout is completed, the limited spaces among the courtyards form lanes. Lanes can also connect courtyards, wading spaces and nodes. And wading spaces and nodes often cause partial organizational changes in courtyards or lanes. Therefore, the prototypes of the organizational structure of the four elements all influence each other.

From the perspective of the courtyard layout, it may be clustered or scattered. In flat area, the large amount of fertile land makes the arable land relatively large, so the layout of the courtyard is clustered. On the other hand, in valleys, gentle slopes, and steep slopes, the layout of courtyards can be linearly scattered or dotted scattered according to the locations of farmland. In this way, most of the courtyards are distributed next to the farmland, ensuring the efficiency of farmland management. The Chinese have attached great importance to Feng Shui since ancient times, which has been rooted in all aspects of rural planning. Feng Shui is an empirical discipline that studies the environment and the rules of the universe, which emphasizes that man belongs to nature, and nature belongs to man, too. These concepts make the villagers think highly of lighting, at the same time make the courtyard facing the waters and with their back to the mountain.

The lanes are mostly gaps among the farmhouses. The structure organization of lanes leads to the close proximity of farmhouses, which makes it easier to visit relatives and friends, help each other, and bargain. At flat areas, the lanes among the clustered courtyards are often organized to be network-shaped. The linearly scattered courtyards are often connected by tree-shaped lanes. The main lane likes the "trunk", parallel to contour lines and along the valley or slope. The secondary lanes along the mountain are connected to the courtyard, similar to the shape of a "trees' branch". Z-shaped lanes are connected the dotted scattered courtyards on steep slopes, winding like the " $Z$ " shape (as shown in Fig. 5).

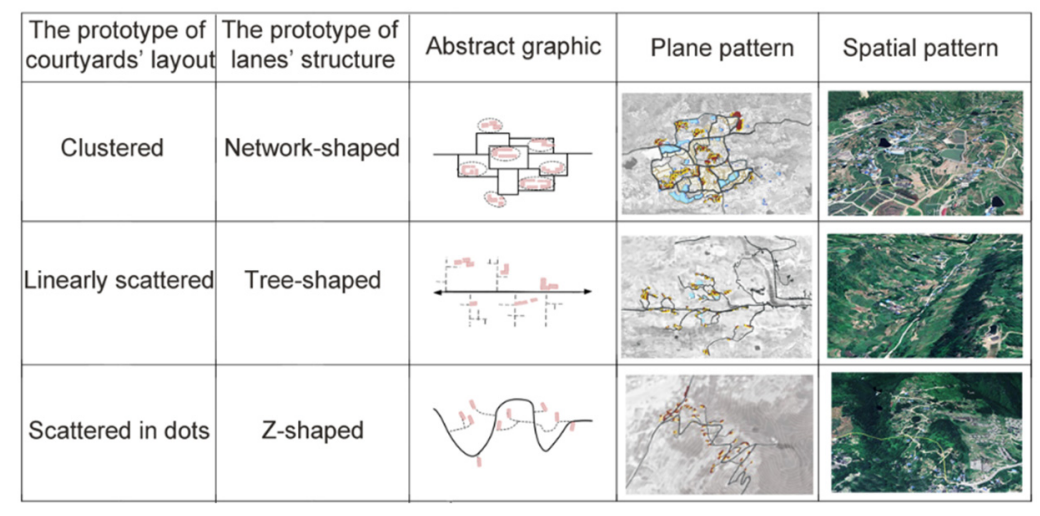

Figure 5: The prototype of courtyards' layout and lanes' structure. 
The structural organization of courtyards and lanes is usually affected by wading spaces and nodes. Wells and village entrances often cause partial expansion of courtyard layouts or lanes' structures. Larger spaces (such as ponds) will also change the layout and expand the sense of sequence of the public space. Streams and water channels are affected by the terrain, and the layout of courtyards and lanes is often consistent with the direction of the water flow of the streams or water channels, thereby forming a series of courtyard clusters or cul-de-sac.

\subsubsection{Decoration and function}

Villagers pay more attention to regional conditions when constructing public spaces in Jinyun Mountain. They rely on folk crafts passed down from generation to generation, using local natural resources such as "bamboo, soil, stone, grass" to build walls, pave the ground, make decorations, and so on. Materials such as rubble, rammed earth and bamboo woven with mud are used to build walls; strips of stone, gravel, bricks or the mixture of rammed earth, blue bricks and pebbles are used to pave the ground. In addition, abandoned production or living appliances such as stone benches and millstones are used as rest facilities, flowerpots, and decorations.

Varies activities of public spaces can be divided into necessary activities and spontaneous activities. Daily farming, breeding, lifting water, firewood stacking are necessary activities under agricultural production methods. Therefore, the public space for these activities must still be designed in the resettlement area to meet the needs of daily life. While spontaneous activities often rely on an "excuse". It is not restricted to a specific time, but participates in the activities of others and enjoys it [15]. For example, villagers in Jinyun Mountain often start chatting on the walk, and decide to play cards after chat. These activities are impromptu. Therefore, specific event facilities are not needed in many public spaces, but public spaces must be accessible to people.

\subsection{Translation of each element}

The elements of rural public space include lanes, courtyards, wading spaces and nodes, and their translation requires full consideration of the willingness of the migrants. According to the method of typology, the three layers of structure organization, plane shape, function and decoration of each element were translated.

\subsubsection{Willingness of migrants}

The eco-migration resettlement area is close to Jinyun Mountain Scenic Area, which has beautiful scenery and can develop tourism industry. Migrants are eager to adapt to a new life and earn more income. The willingness of the migrants and the tourism resources of the resettlement area were integrated into the development mode of the ecotourism industry. This will not destroy the environment of the resettlement area, but also increase the sense of belonging of the villagers.

\subsubsection{Strategies for translating prototypes}

The third typology pointed out that typology is not to repeat the past prototype form, but to assemble the components of each element according to three meanings. Three meanings correspond to three methods of translation: (a) Directly select the prototype; (b) Transform or reorganize the prototype; and (c) Add the new types [16].

In order to meet the actual needs of development and villagers' lives. We need to add a ring road to carry vehicle traffic. In this case, we must know that the development of the eco-migration resettlement area under the guidance of the design will inevitably change from 
"there are farmhouses and then lanes" to "there are streets and then farmhouses". However, the translation of the overall lane structure also needs to follow the original layout rules. Combined with the ring road, the original tree-shaped prototype was transformed into a tree-net structure. Secondly, different layers of lanes were translated by transforming prototypes and adding new types. The prototype of the lane with the same width and proportion as the current main lane was selected to be applied to the main lane. The types of activities on the main lane were supplemented to make it lively and full of different scenery. The prototype with $\mathrm{D} / \mathrm{H} \approx 1$ and a width of $3-5 \mathrm{~m}$ was selected for the secondary lane. The prototype with $\mathrm{D}<3$ was selected for path. The path must take into account the needs of the migrants and the terrain. All the walls of the lanes are made of gravel, and the ground is made of rammed earth, bricks and pebbles.

On the basis of retaining the original characteristics of the courtyard, the economic and service value of the courtyard must be attached. When the courtyard was being laid out, its distance from the farmland must be considered. Based on the resettlement capacity requirements and the location of the farmland, the prototype of the clustered layout of courtyards was selected, which not only saves the farmland area but also conforms to the tree-net structure of lanes. Secondly, considering the tourism development needs and migrants' willingness, the courtyard was divided into two types: tourism residential type and service type. The tourism residential type was selected as "single closed" and "two-sides closed". These prototypes were reorganized into "series connected" and "closed". The series connected type is located on steep terrain. The closed type is located on flat terrain. At the same time, the courtyard area was expanded, its original vegetable fields, ponds and other resources were used to continue self-sufficient life, and catering, chess and card activities were increased to enhance its economic value. Some of the original courtyards were transformed into service courtyards. The original plane shape of the courtyard was preserved and divided into multiple spaces to supplement the activity type. For example, the village reading room was divided into a space to serve as a tearoom (as shown in Fig. 6). In addition, villagers were encouraged to participate in the design together.

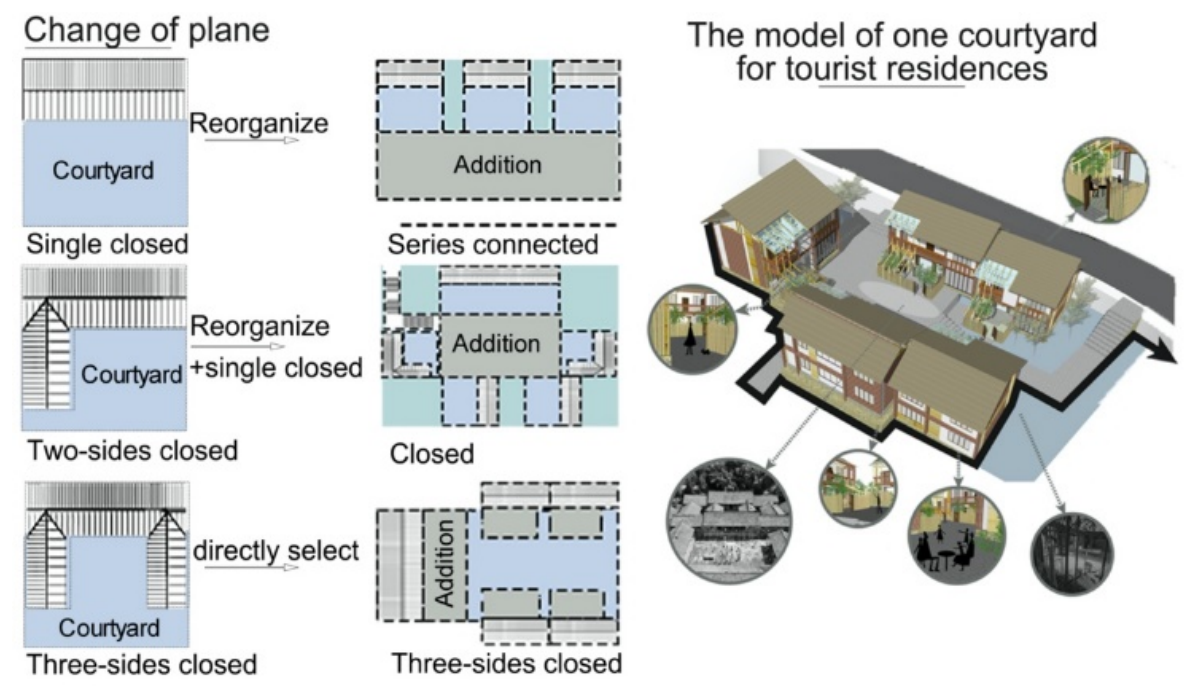

Figure 6: The process of translating prototypes of courtyards for tourist residences. 
The construction of wading spaces emphasizes on conforming to the terrain and building according to the local material, and pursuing the beauty of nature and original. Therefore, based on hydrological analysis, the water was connected the village, connecting the existing streams and canals of village, platforms and boardwalks were added with specific functions to increase the possibility of activities. Some abandoned wells were used to create farming cultural landscapes to let tourists experience rural lifestyle.

In order to further improve the sense of belonging of migrants, the nodes need to be supplemented. The large trees and stone monument on the original site were used to mark the entrance of the village. Beside these signs, local bamboo was planted to improve the village entrance landscape. In addition, vacant land was used to supplement node types. Through consultation with migrants, the vacant land was renewed as a fitness area, children's activity area, etc. These areas allow villagers to participate in the graffiti of the walls when decorating, thus creating some storytelling scenes.

\section{GENERATION OF PUBLIC SPACE SYSTEM}

The village public space system is generated by superimposing each single element which is translated. Because of its systematic and integral nature, the point should be on combining and optimizing the connection between lanes, courtyards, wading spaces and node spaces to make the public space system more reasonable (as shown in Fig. 7). For example, the structure of lanes is adjusted to suit courtyards, wading spaces and nodes. Therefore, in order to achieve the rationality of the public space system and maximize the overall benefits, we first optimized and adjusted the individual elements, and then superimposed the optimized individual elements. In this way, we can further guide the design plan of the public space in the eco-migration resettlement area to be refined.

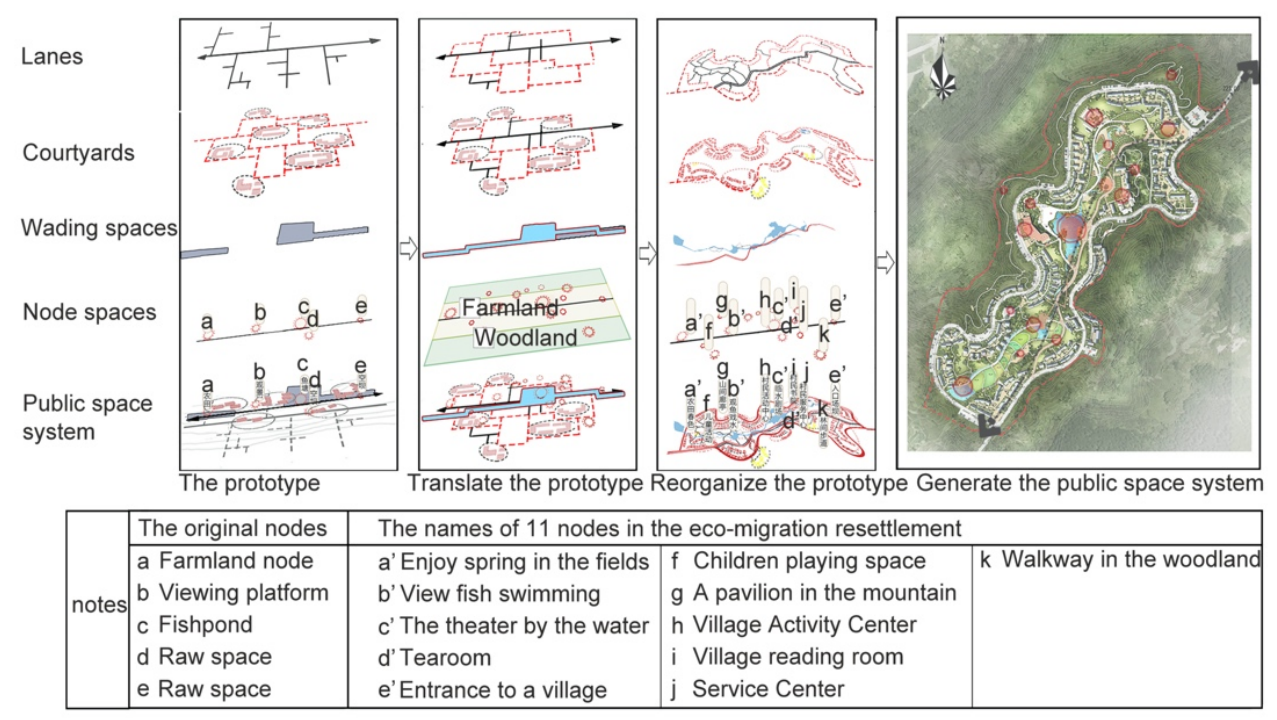

Figure 7: Generating a process for a design scheme of public space for eco-migration rural resettlement. 


\section{CONCLUSION}

As a spiritual place for villagers in their daily life, rural public spaces accumulate unique historical characteristics and cultural customs of each village. For eco-migration resettlement areas, the remoulding of rural public spaces is particularly important. The construction of rural public space in China often stems from China's long traditional culture, which involves landform features, historical habits, feng shui, and so on. This article takes an eco-migration resettlement area in the mountainous areas of western China as a research object and uses a new typological method to design its rural public space. This method extracts and analyses the prototype of the rural public space, and designs the rural public space of the eco-migration resettlement area according to the development needs of the resettlement area and the original lifestyle of the villagers. This is also the analysis and thinking of rural public space planning under eco-migration, and it is worthy of reference.

\section{ACKNOWLEDGEMENTS}

The authors would like to acknowledge the Prof. Jie Zhu for his guidance on this research. We also appreciate $\mathrm{PhD}$ candidate Li Tan for his valuable suggestions on this article.

\section{REFERENCES}

[1] Zhou, L. \& Ruomei, S., Study on the correlation between ecologically sensitive zone and poor area. Rural Economy and Society, 5, pp. 49-56, 1994.

[2] Zhou, L. \& Ruomei, S., Study on the Relationship between Development Mode and Ecological Change of Poor Mountain Areas in China, Shanxi Economics Press: Taiyuan, pp. 1-25, 1997.

[3] Fengrui, L., Ecological Migration: The fundamental plan of environmental construction in western China. China Economic News, 31, pp. 15-16, 2001.

[4] Greenpeace in Beijing, Climate Change and Poverty: A Case of China. http://www.doc88.com/p-2942078179136.html. Accessed on: 14 Mar. 2014.

[5] Zhiming, B., On the definition, classification and some questions of ecological migration. Journal of Minzu University of China, 1, pp. 27-31, 2006.

[6] Cunhai, Y., Discussion on ecological poverty, ecological resettlement and community integration in western. Inner Mongol Social Science, 1, pp. 128-133, 2004.

[7] Colquhoun, A., Postmodernism and structuralism: A retrospective glance. Assemblage, 5, pp. 7-15, 1988.

[8] de Quincy, Q., Dictionnaire historique d'architecture, le Clere, pp. 629, 1832.

[9] Argan, G.C.., On the typology of architecture. Architectural Design, 12, pp. 564-565, 1963.

[10] Krier, R., Urban Space. Rizzoli, pp. 8-59, 1993.

[11] Kelin, S., Typology and Urban Morphology, China Building Industry Press: Beijing, pp. 38, 2010.

[12] Lijun, W. \& Ping, S., Typology Architecture, Tianjin: Tianjin University Press, pp. 2846, 2004.

[13] Beibei District Chengjiang Town people's government, Records of the Chengjiang Town, Beibei Shuanghe Press: Chongqing, pp. 150-496, 2005.

[14] Changjiang Gao., Local Feeling, Local Customs, Local Accent - The Language of Chinese Rural Culture, Jilin University Press: Jilin, pp. 165, 1994.

[15] Min, J. \& Wentong, H., Composition and contemporary evolution of the public space system of traditional Villages: A case study of Banliang Village. Settlement, 5, pp. 4954, 2019.

[16] Shenning, S., Review typology. Architect, 6, pp. 5-19, 2006. 